\title{
Electrochemical detection of oxacillin resistance with SimpleStat: a low cost integrated potentiostat and sensor platform
}

Received 00th January 20xx, Accepted 00th January 20xx DOI: $10.1039 / x 0 \times x 00000 x$

\begin{abstract}
Adrian Butterworth, ${ }^{a}$ Damion K Corrigan ${ }^{a}$ and Andrew C Ward*a
Testing outside the laboratoty environment, such as point of care testing, is a rapidly evolving area with advances in the integration of sample handling, measurement and sensing elements widely reported. Low cost, simple to use systems are important in this context because they provide a route to devices that can be used outside the laboratory and could be implemented in low resource settings where advanced diagnostic testing is often unavailable. Here, we present an open source highly simplified electrochemical platform, called SimpleStat, that has been programmed to perform differential pulse voltammerty and can be used to detect the presence of OXA-1 DNA sequences for oxacillin resistance. This DNA sensor can be used to specifically detect the presence of the OXA-1 gene, contrasted to the tetA gene which encodes for tetracycline resistance. These measurements were performed with both polycrystalline gold electrodes as a benchmark and electrode integrated into the SimpleStat printed circuit.
\end{abstract}

\section{Introduction}

Sensor systems are playing an increasingly important role in a wide range of scenarios, including industrial, environmental and healthcare detection challenges ${ }^{1-6}$. In particular Antimicrobial resistance (AMR) is one of the greatest threats to modern medical practice and there is a strong imperative to reduce and better control the use of antimicrobial drugs, known as antimicrobial stewardship, to slow the increase in prevalence of AMR pathogens ${ }^{7,8}$. Diagnostic testing plays an important role in this, offering the ability to rule in/rule out the use of antimicrobial drugs, thus supporting antimicrobial stewardship and increasing the length of time existing drugs are useful ${ }^{9}$. Current challenges to the widespread uptake of diagnostic tests include significant barriers to market (such as high development costs and regulatory frameworks), a sensor and platform device that can be used across a large range of operational scenarios (including, medical, agricultural and environmental) and is usable by non-experts without the need for advanced laboratory infrastructure ${ }^{10,11}$. Electrochemical sensors and instrumentation could address these challenges and help to enable a paradigm shift in the way that pathogenic organisms are identified.

A number of low cost, open source potentiostats have been developed in recent years. The CheapStat was proposed as a

a. Department of Biomedical Engineering, Wolfson Centre, 106 Rottenrow, University of Strathclyde, Glasgow, UK, G4 ONS.

Electronic Supplementary Information (ESI) available: [details of any supplementary information available should be included here]. See DOI: 10.1039/x0xx00000x low cost platform and educational potentiostat and was demonstrated with the detection of electroactive substances including ascorbic acid in orange juice and $\mathrm{N}$-acetyl-paraaminophenol ${ }^{12}$. The accuracy of open source potentiostats was greatly improved by the creation of the DStat, which provides a high end measurement platform comparable to a commercial instrument, combined with an easy to use interface ${ }^{13}$. Several others have taken inspiration from these studies, including the development of an instrument based upon a programmable circuit on a chip ${ }^{14}$ and a wireless electrochemical platform for use with a smartphone ${ }^{15}$. Two separate studies also explore the use of the Texas Instruments LMP91000 potentiostat chip, which could be used to reduce circuit complexity ${ }^{16,17}$. Instruments have also been produced with simplified circuits, such as the SIMS integrated electronic system, incorporating organic and silicon electronics for simplified electrochemical measurements ${ }^{18}$. The common theme across all these instruments is that they focus upon accurate, low cost electrochemical measurement for a wide range of electrochemical needs. A detection device based upon technologies such as these could help to reduce some of the barriers described above.

OXA type $\beta$-lactamases are widespread in Gram negative bacteria, and can provide resistance to $\beta$-lactam based antibiotics through cleavage of the active $\beta$-lactam motif. OXA-1 $\beta$-lactamases are resistant to the action of $\beta$-lactamase inhibiting antimicrobial drugs. Although OXA-1 exhibits a narrow spectrum of activity, it is frequently associated with the presence of other extended-spectrum $\beta$-lactamases (ESBLs), such as those against third and fourth generation cephalosporins, as well as genes conferring resistance to aminoglycosides and fluoroquinolones which further reduces 
treatment options ${ }^{19,20}$. Furthermore, the effectiveness of $\beta$-lactamase inhibitor combination treatments is reduced when both OXA-1 and another ESBL is present in a pathogen, compared with an ESBL alone. The OXA-1 gene is among the most common OXA $\beta$-lactamase ${ }^{21}$, and its prevalence in the population can make effective treatment of infections carrying this gene difficult when combined with other resistance factors. A number of electrochemical measurements have been developed to detect clinically relevant DNA sequences and there are examples of antibiotic resistant DNA sequence detection using lab based systems ${ }^{22-24}$.

In this paper, we explore the use of a highly simplified potentiostat circuit that can be produced at very low cost with the minimum number of components possible. This reduces the cost of the platform and marks a step towards extremely low cost sensor platforms for electrochemical monitoring and detection. Furthermore, we demonstrate that the platform can be used to perform Differential Pulse Voltammetry (DPV) measurements and detect the binding of PCR products from the OXA-1 gene on the electrode surface. We achieve this on both polycrystalline gold electrodes and on gold plated $\mathrm{PCB}$ electrodes integrated onto the board.

\section{Experimental}

\section{Circuit Design and Layout}

A simplified circuit was designed based upon the minimum number of components possible (Figure 1). The ATTiny412 microcontroller (Microchip Technology Inc.) was used to control the circuit and perform analog to digital conversion (ADC) of the results. This microcontroller was selected due to its low cost (<\$1), 8-bit Digital to Analog Converter (DAC), 10-bit ADC, easy to solder package and low pin count (8 pins). It also contains an on-board oscillator, which removes the need for an external crystal further simplifying the device. A bank of gain resistors was included in the circuit design in order to provide flexibility over the current ranges that can be recorded in a measurement. These are manually switched using a DIP switch, to keep device complexity low. A low cost LED is included in the device, so that for very simple measurements, the difference between a positive and negative sample can be easily determined without any external devices. The instrument can also be interfaced to a computer for data logging, via the UART peripheral so that a greater amount of information can be captured. A UART-USB bridge is required to achieve this functionality (e.g. the FT234XD chip from FTDI Ltd).

The analog front end uses two TLC2262 operational amplifiers because of their low input bias current ${ }^{12}$. In order to keep the design as simple as possible, the potentiostat and transimpedance amplifier operational amplifiers are biased at $1.25 \mathrm{~V}$ vs GND using a network of resistors. This provides the ability to hold the electrochemical cell at a potential between $-0.6 \mathrm{~V}$ and $0.6 \mathrm{~V}$. To maximise the accuracy of the complete instrument, two trim potentiometers are included in
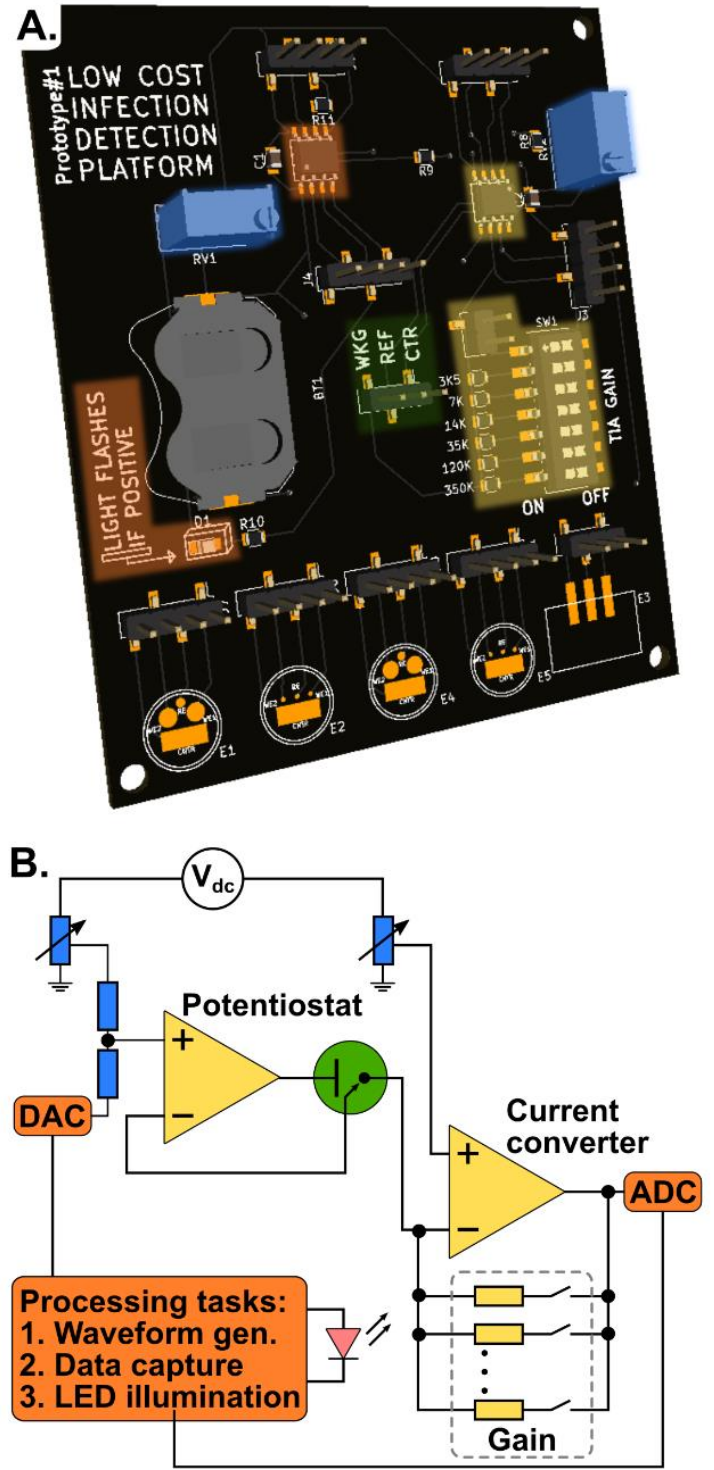

Figure 1: SimpleStat PCB and circuit schematic. (A) The $7 \mathrm{~cm} \times 8 \mathrm{~cm} P C B$, showing the location of the key components. (B) Schematic of the circuit, showing the microcontroller functions (orange), the calibration circuit (blue), the analog is used to manually change the required gain, based upon the anticipated resistance changes.

the design so that the $1.25 \mathrm{~V}$ zero point can be calibrated prior to the start of a measurement.

The printed circuit was designed in the open source software package KiCAD, which was also used to design two simplified electrode patterns, integrated onto the board and used for detection of the OXA-1 gene. Gold coated Printed Circuit Boards (PCBs) are readily available from a wide range of manufacturers and were used in this design in order to yield a gold electrode surface for sensing and detection. The microcontroller firmware was produced in Atmel Studio, using Atmel Start to achieve the basic chip configuration. (See supplementary material for copies of the design files, firmware and device Bill of Material). 


\section{Plasmid, primer design and PCR}

Two electrode systems were explored in this study: Polycrystalline gold electrodes (PGEs) with a diameter of $2 \mathrm{~mm}$ (IJ Cambria) and the integrated on board electrodes ( 2 and 0.5 $\mathrm{mm}$ discs). All solutions were prepared with deionised water (di $\mathrm{H}_{2} \mathrm{O}$, Scientific Laboratory Supplies). Polymerase Chain Reaction (PCR) was performed using a Hst+ DNA polymerase and associated reagents which were obtained from Qiagen. 3Mercapto-1-propanol (MCP) was obtained from Sigma Aldrich. All other chemicals were purchased from Acros Organics.

PCR, performed according to the instructions provided with the reagents, was used to amplify an initial $344 \mathrm{ng}$ of template for 30 cycles (Figure $2 \mathrm{~A}$ and $2 \mathrm{~B}$ ). The template consisted of a custom plasmid extracted from transformed $E$. coli $\mathrm{DH} 10 \beta$ cells. This plasmid harbours inactive segments of several plasmidborne antibiotic resistance genes, including the OXA-1 $\beta$ lactamase gene (Figure 2C). Forward (AACAGAAGCATGGCTCGAAA) and reverse (TGGTGTTTTCTATGGCTGAGTT) primers were used to amplify a 116 base-pair section of this template, for OXA-1, which is complementary to the DNA probe sequence immobilised onto the gold surface ([ThiolC6]AACAGAAGCATGGCTCGAAA).

\section{Electrode Functionalisation \& Assay Conditions}

The polycrystalline gold electrodes used for benchmarking were cleaned by immersing in Piranha solution for 10 mins, followed by polishing with alumina slurry and sonication in deionised water for 10 mins. The polished electrodes were then cleaned by cyclic voltammetry in $0.1 \mathrm{M} \mathrm{H}_{2} \mathrm{SO}_{4}$ between $0 \mathrm{~V}$ and $1.7 \mathrm{~V}$ (vs. $\mathrm{Ag}-\mathrm{AgCl}$ ) for 20 cycles. Electrodes were functionalised by immersing in $20 \mu \mathrm{L}$ immobilisation buffer consisting of $0.8 \mathrm{M}$ PBS + $5 \mathrm{mM} \mathrm{MgCl} 2+1 \mathrm{mM}$ Ethylenediaminetetraacetic acid (EDTA) + $5 \mathrm{mM}$ Tris(2-carboxyethyl)phosphine (TCEP), and containing $30 \mu \mathrm{M} \mathrm{MCP}$ and $3 \mu \mathrm{M}$ DNA probe at $30^{\circ} \mathrm{C}$ overnight. Following incubation, electrodes were rinsed in the immobilisation buffer, then in $0.2 \mathrm{M}$ phosphate buffer (PB), $10 \mathrm{mM} P B$ and $10 \mathrm{mM} P B+10 \mathrm{mM}$ EDTA. Finally, the surface was backfilled with $1 \mathrm{mM} \mathrm{MCP}+5 \mathrm{mM}$ TCEP in $\mathrm{diH}_{2} \mathrm{O}$ for one hour.

After backfilling and rinsing in $\mathrm{diH}_{2} \mathrm{O}$, baseline DPV measurements of the SAM layer were taken using a PalmSens PS4 potentiostat (PalmSens) and the SimpleStat potentiostat in $2 \mathrm{mM} \mathrm{Fe}(\mathrm{CN})_{6}^{3-} / \mathrm{Fe}(\mathrm{CN})_{6}{ }^{4-}$ buffered with $50 \mathrm{mM} \mathrm{PB}+200 \mathrm{mM}$ $\mathrm{KCl}(\mathrm{pH} 7)$. All benchmark measurements using the PGEs were recorded in a three-electrode cell, with a platinum foil counter electrode and gold reference electrode. DPV measurements were performed at a range of potentials between $-0.6 \mathrm{~V}$ and $+0.3 \mathrm{~V}$, with a step potential of $10 \mathrm{mV}$, pulse potential of $49 \mathrm{mV}$, pulse time of $50 \mathrm{~ms}$ and scan rate $20 \mathrm{mV} / \mathrm{s}$.

Once baseline measurements were complete, electrodes were rinsed in $200 \mathrm{mM} \mathrm{PB}+800 \mathrm{mM} \mathrm{KCl}$ and then incubated at $30^{\circ} \mathrm{C}$ for one hour with $20 \mu \mathrm{L}$ PCR product which had been heated to $90^{\circ} \mathrm{C}$ to denature the DNA double strand. In order to remove
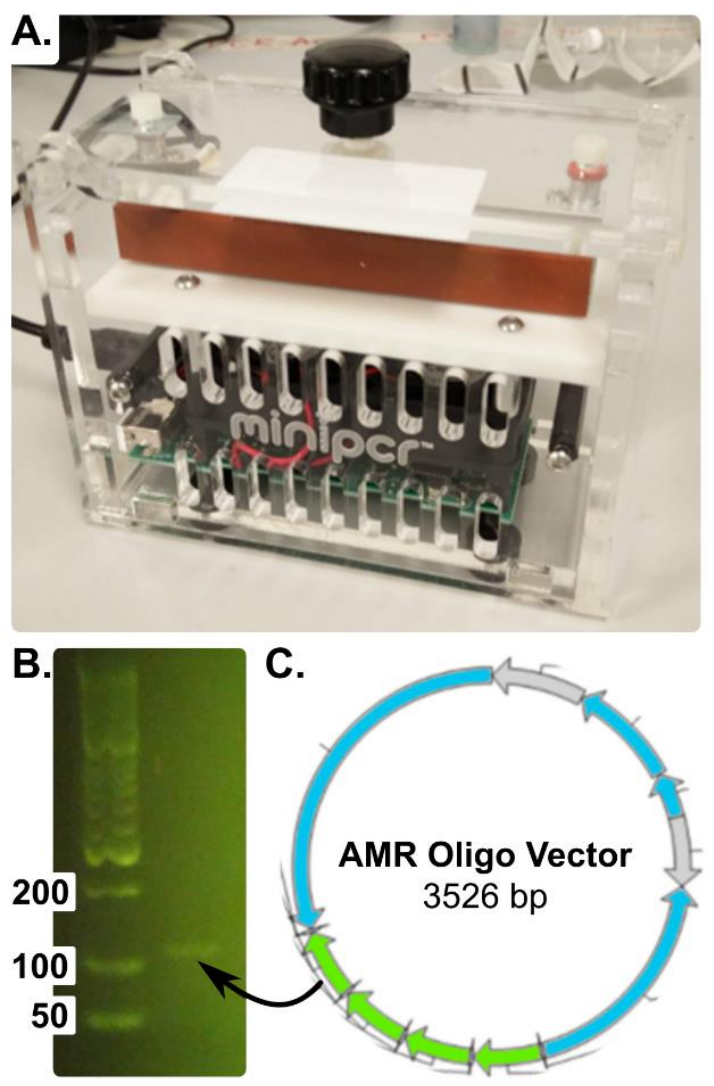

Figure 2: PCR methodology used in this study. (A) Mini PCR machine used for DNA amplification. (B) Agarose gel showing amplification product from the OXA-

unbound DNA and other PCR components each electrode was rinsed with $200 \mathrm{mM} \mathrm{PB}+800 \mathrm{mM} \mathrm{KCl}$ and $50 \mathrm{mM} \mathrm{PB}+200 \mathrm{mM}$ $\mathrm{KCl}$ before the second set of DPV measurements were recorded.

The same preparation protocol was used for functionalisation and measurement of target DNA hybridisation with the on board electrodes. The only difference being the approach to cleaning the surface. For the electrodes sited on the board, gentle rinses with $\operatorname{diH}_{2} \mathrm{O}$ and $0.1 \mathrm{M} \mathrm{H}_{2} \mathrm{SO}_{4}$ were employed prior to immobilisation of the DNA probe strand.

\section{Results \& Discussion}

\section{Comparative redox measurements}

A set of characterisation measurements were performed using the commercial potentiostat. For these measurements, the on board electrodes were not modified with DNA. This was done to demonstrate the satisfactory performance of the on board electrodes for later functionalisation with thiolated ssDNA probe strands.

Three electrochemical measurement techniques were employed for this characterisation using a measurement buffer containing the redox agents potassium ferri-ferrocyanide. The measurement techniques used were cyclic voltammetry (CV), electrochemical impedance spectroscopy (EIS) and differential pulse voltammetry (DPV). The CV results (Figure $3 \mathrm{~A}$ ) show the 
expected behaviour with a reversible CV (peak separation: $83 \mathrm{mV}$ ) and with sweep rate dependence centred around $0 \mathrm{~V}$
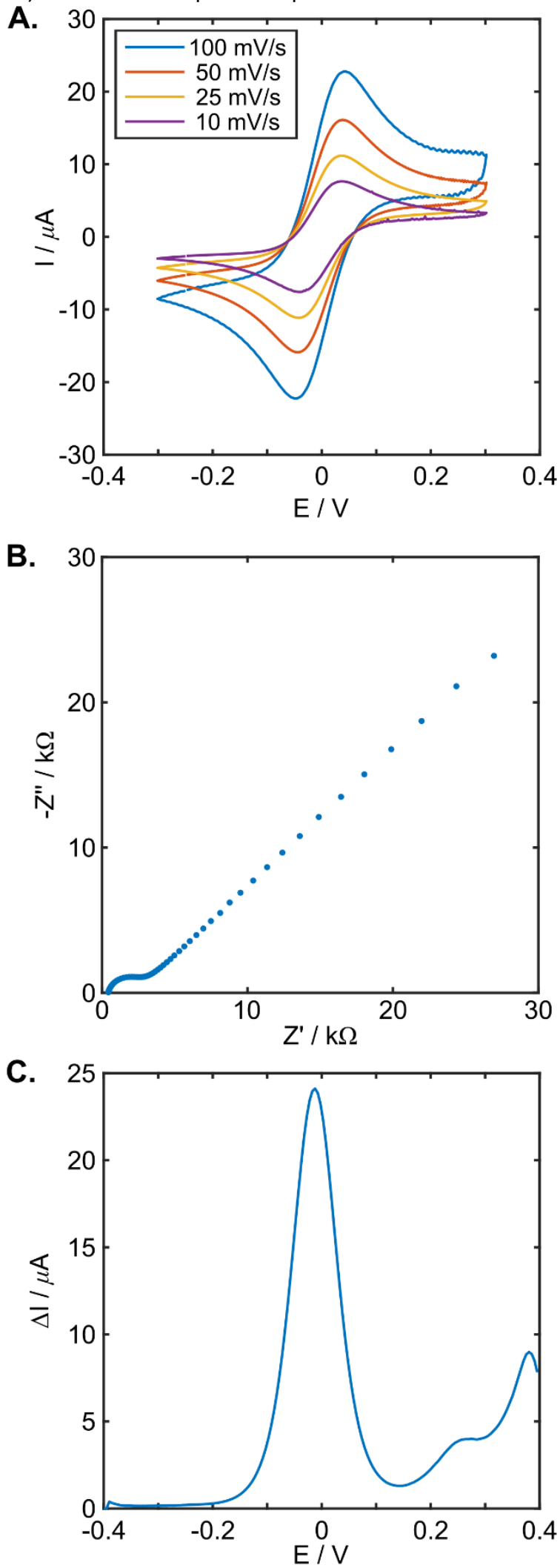

Figure 3: Characterisation of the SimpleStat on board electrodes carried out with a commercial potentiostat. (A) CV, (B) EIS (B) and (C) DPV. Measurements recorded in $2 \mathrm{mM}$ potassium ferri-ferrocyanide $+50 \mathrm{mM}$ phosphate butfe
$200 \mathrm{mM} \mathrm{KCl}$. A gold reference electrode was used for all measurements. due to the use of a gold electrode as the reference electrode in the on board cell.

The Randles-Sevcik equation (1) was used to calculate the geometric surface area of the electrode:

$$
i_{P}=0.4463 n F A C\left(\frac{n F v D}{R T}\right)^{1 / 2}
$$

Where $i_{P}$ is the peak current, $n$ is the number of electrons transferred, $A$ is the electrode area, $C$ is the redox species concentration, $F$ is Faraday's constant, $v$ is the sweep rate, $D$ is the diffusion coefficient, $R$ is the universal gas constant and $T$ is the temperature. This showed that the active electrode area was $65 \%$ greater than the geometric area. This calculation along with the expected appearance of the CV plots gave confidence that the electrodes on the SimpleStat board were viable for the DNA detection assay.

In addition, EIS experiments were performed in order to further characterise the electrode (Figure 3B) and it was evident that the expected EIS response was present. The semi-circular region of the plot is indicative of a charge-transfer process $\left(R_{C T}\right)$ and the $45^{\circ}$ line indicative of linear diffusion $(W)$. After fitting to the established Randles' equivalent circuit 25 a value for $R_{C T}$ of $233 \Omega$ was determined. This was verified by calculating the theoretical value for $R_{C T}$ using the thermodynamic expression (2):

$$
R_{C T}=\frac{4 R T L}{n A D F^{2} c}
$$

Where $L$ is the diffusion length and other symbols are as per (1). The calculated value for $R_{C T}$ was $293 \Omega$ which is close to the measured value of $233 \Omega$. The discrepancy between the measured and calculated $R_{C T}$ value is most likely explained by the error associated with estimating the diffusion length $L$.

The DPV curve (Figure 3C), also showed the expected response with a clear current peak centred around the mid-potential for the redox couple. Again, as with the $\mathrm{CV}$ s, this peak is positioned over $0 \mathrm{~V}$ due to the use of a gold electrode for the reference.

The basic electrochemical characterisation shown in this section gave confidence that the three electrode systems on board the Simplestat could serve as a basis for the DNA detection assay.

\section{SimpleStat design and cost}

The SimpleStat device was designed with a strong focus upon overall cost, whist maintaining sufficient functionality to perform a DPV measurement. Ultimately, the instrument is designed to function in a standalone manner, with the in-build LED indicating the test results (e.g. on for a positive sample, off for a negative sample). The total component cost to build the instrument (including PCB printing with the four integrated electrodes) was less than $f 10$ per board. At higher volumes, we 
estimate this would drop to less than $£ 3.50$ per board. The board microcontroller was programmed using the Atmel Studio suite of software to perform DPV measurements taking advantage of the DAC on the board to produce the required step/pulse waveform (Figure 4A) and to capture the current response (Figure $4 B$ ) using the ADC. The DPV parameters used in our experiment were found to yield measureable changes in the peak current observed at the electrode surface. Further optimisation of these results could be achieved by adjusting these parameters including exploration of Square Wave Voltammetery (SWV).

Although the SimpleStat performed well throughout the measurements described below, one limitation of the current device relates to the dependence on consistency of the power supply. Throughout the testing performed, $3 \mathrm{~V}$ lithium cell was used without a voltage regulator to ensure a fixed voltage supply. This was done in order to keep the cost and power requirements of the board low, by avoiding the use of a separate voltage regulator circuit. This has a key drawback because changes in the potential of the lithium cell (for example, as it discharges) will affect the calibration of the Simplestat and thus accuracy of measurements. The lithium cell used in this study had a near full charge and therefore we did not experience serious problems with this limitation. However, in the future, the SimpleStat could be enhanced with a simplified constant voltage circuit using Zener diodes.

\section{Simple Stat vs a benchmark potentiostat for OXA-1 detection}

A series of measurements were carried out with the SimpleStat and a commercially sourced potentiostat, with the PGEs prepared as detailed above. These served as a benchmark to compare the measurement accuracy of the SimpleStat. The DNA detection strategy is based upon an OXA-1 complementary probe on the surface of the electrode. When the OXA-1 DNA is present, a change in the Faradaic current passed across the electrode surface is expected, as the negatively charged $\mathrm{Fe}(\mathrm{CN})_{6}{ }^{3-} / \mathrm{Fe}(\mathrm{CN})_{6}{ }^{4-}$ is held away from the electrode surface by the negatively charged OXA-1 DNA bound to the probe (Figure $5 A)$.

The electrode surface was prepared to bear a simple $\mathrm{MCH}$ single stranded DNA (ssDNA) probe monolayer. The DNA probe sequence is specific for OXA-1. At this stage, the DPV peak current (brown curve) is higher and this is because of the greater access the redox couple has to the electrode surface. Upon binding of the OXA-1 PCR product, surface access is hindered both physically through surface blockage and electrostatically through repulsion between the DNA duplex and the negatively charged redox reporter. This is a wellestablished principle for electrochemical DNA detection employed previously ${ }^{22}$. These results show a good degree of equivalence between the commercially available potentiostat and the SimpleStat (Figure 5B). After hybridisation of the OXA1 probe, a clear decrease in the peak current magnitude can be detected for both the commercial potentiostat system and the
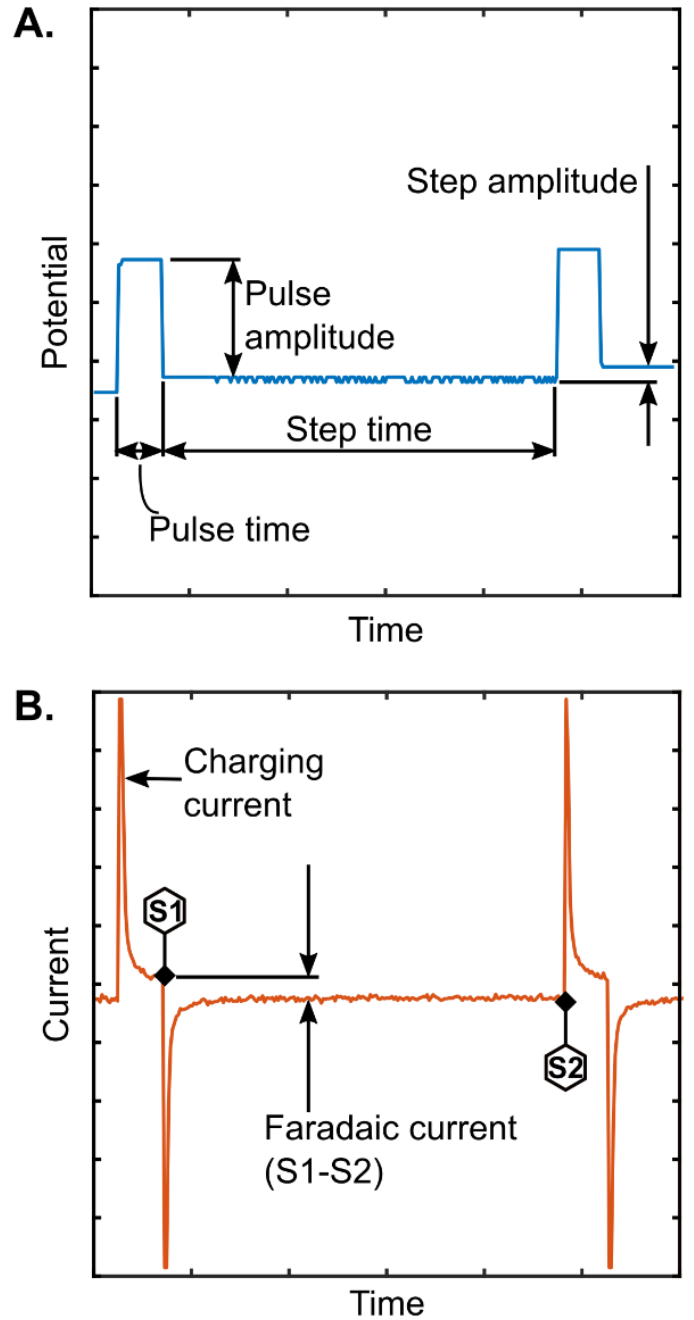

Figure 4: Raw current waveforms resulting from a DPV measurement, showing key features. (A) A series of potential pulses are applied to the working electrode using the ATtiny 412 DAC, and are defined by pulse amplitude, step amplitude, pulse time and step time. (B) The effect in terms of current across the electrode can be broken down into the double layer charging current immediately after application of the pulse; decaying to the Faradaic current By sampling the current at S1 and S2 using the ATtiny 412 ADC, the net Faradaic charging current.

SimpleStat. The mean drop in the signal upon binding of the OXA-1 DNA to the electrode surface further indicates that a decrease in the magnitude of the signal is observed (Figure $5 \mathrm{C}$ ). This result is encouraging because, whilst the measurement is not identical between instruments, the Simplestat follows the same trend as the commercial potentiostat and would be sufficient for low cost DNA assays such as the one described here.

\section{Use of the SimpleStat on board electrode to detect OXA-1}

To explore the accuracy of the SimpleStat on board electrodes, measurements were performed with PCR product from OXA-1 and tet $A$ (i.e. a different gene encoding tetracycline resistance). Both PCR products were amplified from the same plasmid and detected using the on board electrodes. These measurements, performed with a commercial potentiostat, show that a reduction in the magnitude of the DPV peak occurs as a result of the binding of OXA-1 DNA to the probe on the electrode 
A. 1. Electrode is functionalised with DNA probe SAM. Peak current from redox mediator in absence of OXA-1 DNA.

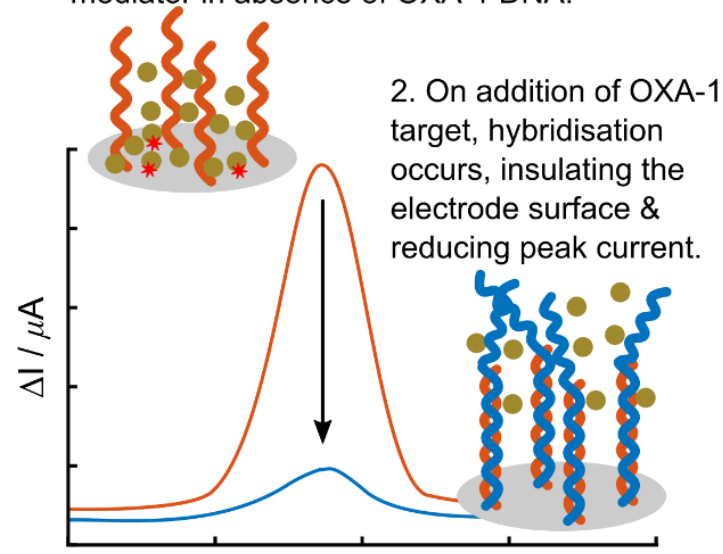

$\mathrm{E} / \mathrm{V}$
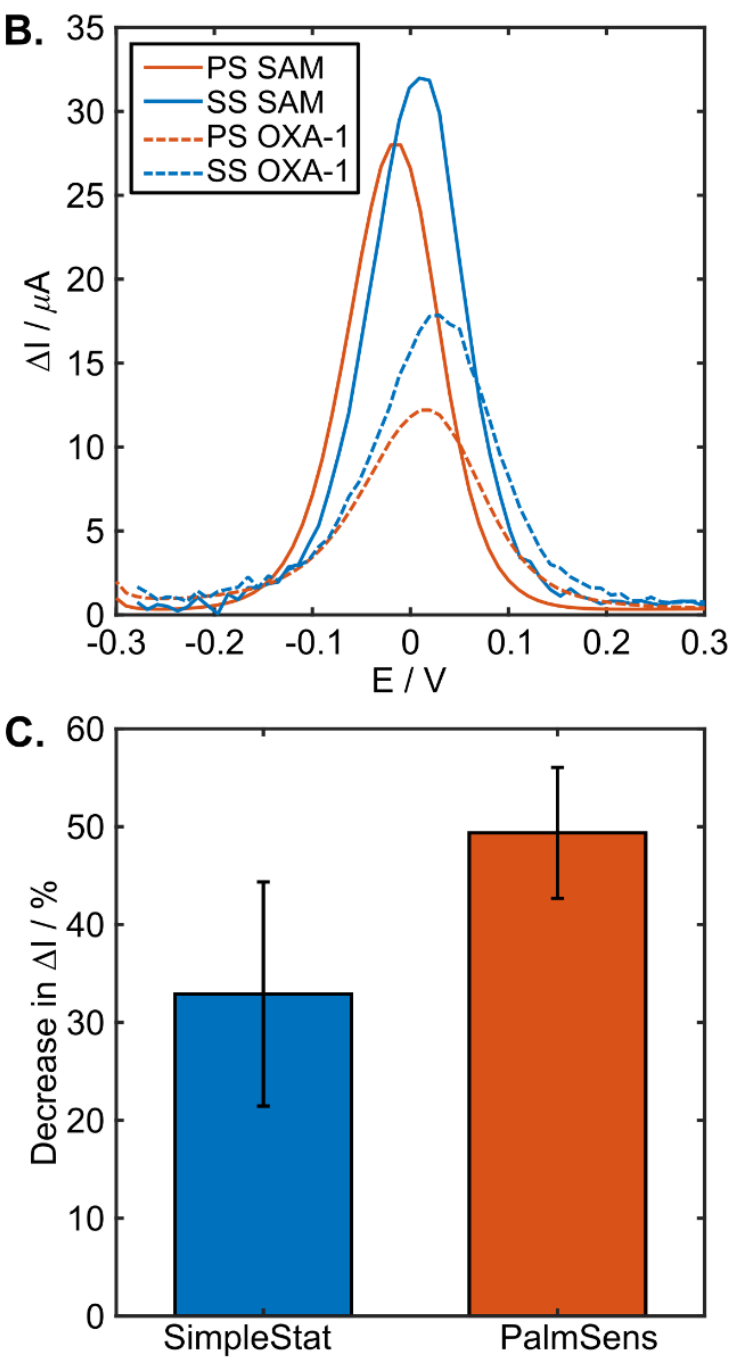

Figure 5: Detection of OXA-1 binding on PGE electrodes. (A) Depiction of the DNA binding process and DNA measurement. (B) Example DPV measurements recorded using the Simplestat and a commercialy available potentiostat and (C) mean DPV peak suppr

surface (Figure 6A). Furthermore, when the tetA PCR product is placed on the electrode, there is negligible signal suppression, which indicates a lower rate of DNA hybridisation (Figure 6B).
This shows that the DNA probe on the electrode surface is specific for OXA-1 and is consistent with previously reported research within our group ${ }^{26}$. The result here therefore provides further confirmation of the specificity between the OXA-1 probe and PCR product. Additionally, the findings with PGEs give confidence that the Simplestat could be used to make clinically relevant measurements, particularly involving PCR product. Detection of a biologically relevant DNA target using the SimpleStat is encouraging and represents an important step towards a fully integrated instrument and sensor for the identification of antimicrobial resistance genes such as OXA-1.

The on-board electrodes were challenging to use because of the very thin layer of gold covering the highly reactive copper surface of the electrodes. Although these results demonstrate the principle that the integrated electrodes could be used for the detection process, a thicker gold layer would be a considerable benefit and will be investigated in future work. This could be achieved by working with a manufacturer to plate a thicker layer of gold during the PCB production process, or through in house electroplating downstream of the $P C B$ production. In addition to increasing the thickness of the gold layer on the surface of the electrode, gold nanoparticles could be explored in future work, by immobilising the DNA to the surface of the nanoparticles and using this in conjunction with a carbon electrode ${ }^{27}$.

By combining the electroanalytical approach adopted here with a PCR reaction, it has been possible to obtain high specificity for the OXA-1 gene and due to the exponential nature of the PCR process high levels of detectable product negated the need for complex electrode modifications often employed to achieve high sensitivity. Future focus will be placed upon migrating the PCR reaction used from a standalone instrument to an integrated PCR performed on the instrument board.

\section{Conclusions}

This study demonstrates how a low cost highly simplified potentiostat system can be used to detect the presence of DNA produced by organisms resistant to the $\beta$-lactam antibiotic drugs. Using DPV measurements we show detection of the OXA1 gene with both PGEs and integrated electrodes on the SimpleStat board. Moving forward, the SimpleStat board will be used to detect a wider range of analytes (including other DNA targets, proteins and related microbial ligands). For DNA detection, we will investigate the use of on board PCR in order to further streamline the measurement process to move towards a device which is usable by non-experts. We anticipate this leading to a device applicable to a range of electroanalytical measurement challenges, particularly those required for pathogen detection in resource limited scenarios where robust and simple assays can have high clinical impact.

\section{Conflicts of interest}



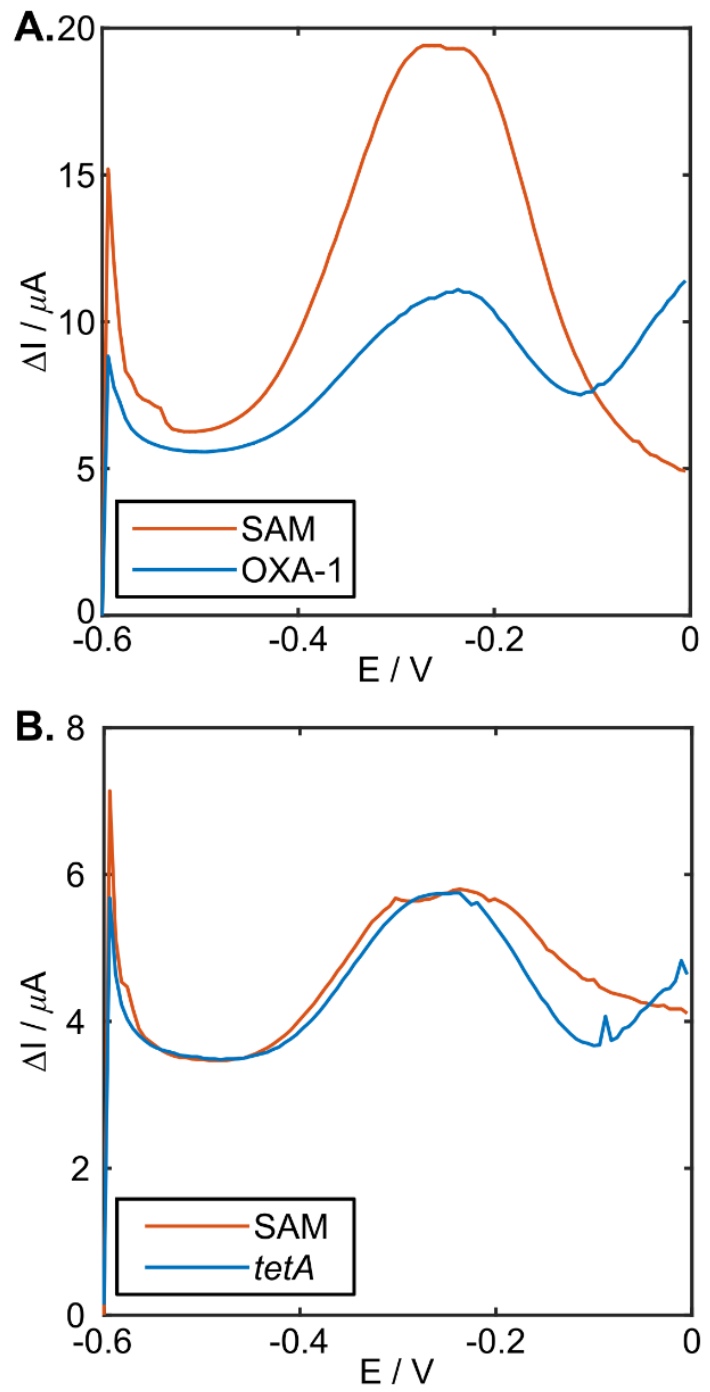

Figure 6: OXA-1 detection using the SimpleStat on board electrodes. (A) Binding of the OXA-1 DNA to the SAM results in a decrease in the current magnitude. (B) A control sample containing DNA from the tetA gene does not bind to the OXA-1 DNA probe, demonstrating the specificity of the probe.

There are no conflicts to declare.

\section{Acknowledgements}

DC \& AW would like to thank the Carnegie Trust for the Universities of Scotland for the Research Incentive Grant which funded this work. $A B$ was funded through a University of Strathclyde PhD scholarship.

\section{Notes and references}

1. Hayat A, Marty J. Disposable Screen Printed Electrochemical Sensors: Tools for Environmental Monitoring. Sensors. 2014 Jun 13;14(12):10432-53.

2. Hulme J. Recent advances in the detection of methicillin resistant Staphylococcus aureus (MRSA). BioChip J. 2017 Jun 1;11(2):89-100.
3. Justino $\mathrm{ClL}$, Freitas $\mathrm{AC}$, Pereira R, Duarte $\mathrm{AC}$, Rocha Santos TAP. Recent developments in recognition elements for chemical sensors and biosensors. TrAC Trends in Analytical Chemistry. 2015 May;68:2-17.

4. Neethirajan S, Tuteja SK, Huang S-T, Kelton D. Recent advancement in biosensors technology for animal and livestock health management. Biosensors and Bioelectronics. 2017 Dec 15;98:398-407.

5. Singh R, Mukherjee MD, Sumana G, Gupta RK, Sood S, Malhotra BD. Biosensors for pathogen detection: A smart approach towards clinical diagnosis. Sensors and Actuators B: Chemical. 2014 Jul 5;197:385-404.

6. Svítková J, Ignat T, Švorc L', Labuda J, Barek J. Chemical Modification of Boron-Doped Diamond Electrodes for Applications to Biosensors and Biosensing. Critical Reviews in Analytical Chemistry. 2016 May 3;46(3):248-56.

7. O'Neill Report. Tackling Drug-Resistant Infections Globally: Final Report and Recommendations. 2016 May. (The Review on Antimicrobial Resistance Chaired by Jim O'Neill).

8. Boucher HW, Talbot GH, Bradley JS, Edwards JE, Gilbert D, Rice LB, et al. Bad Bugs, No Drugs: No ESKAPE! An Update from the Infectious Diseases Society of America. Clin Infect Dis. 2009 Jan 1;48(1):1-12.

9. Ayukekbong JA, Ntemgwa M, Atabe AN. The threat of antimicrobial resistance in developing countries: causes and control strategies. Antimicrobial Resistance \& Infection Control. 2017 May 15;6(1):47.

10. Andreescu S, Sadik OA. Trends and challenges in biochemical sensors for clinical and environmental monitoring. Pure and Applied Chemistry. 2004 Jan 1;76(4):861-78.

11. Sin ML, Mach KE, Wong PK, Liao JC. Advances and challenges in biosensor-based diagnosis of infectious diseases. Expert Review of Molecular Diagnostics. 2014 Mar;14(2):225-44.

12. Rowe AA, Bonham AJ, White RJ, Zimmer MP, Yadgar RJ, Hobza TM, et al. CheapStat: An Open-Source, "Do-ItYourself" Potentiostat for Analytical and Educational Applications. PLoS ONE. 2011 Sep 13;6(9):e23783.

13. Dryden MDM, Wheeler AR. DStat: A Versatile, Open-Source Potentiostat for Electroanalysis and Integration. PLOS ONE. 2015 Oct 28;10(10):e0140349.

14. Lopin P, Lopin KV. PSoC-Stat: A single chip open source potentiostat based on a Programmable System on a Chip. PLOS ONE. 2018 Jul 25;13(7):e0201353.

15. Ainla A, Mousavi MPS, Tsaloglou M-N, Redston J, Bell JG, Fernández-Abedul MT, et al. Open-Source Potentiostat for Wireless Electrochemical Detection with Smartphones. Anal Chem. 2018 May 15;90(10):6240-6. 
16. Aznar-Poveda J, Antonio Lopez-Pastor J, Garcia-Sanchez A-J, Garcia-Haro J, Fernandez Otero T. A COTS-Based Portable System to Conduct Accurate Substance Concentration Measurements. Sensors. 2018 Feb;18(2):539.

17. Bezuidenhout $P$, Smith $S$, Joubert T-H. A Low-Cost InkjetPrinted Paper-Based Potentiostat. Appl Sci-Basel. 2018 Jun;8(6):968.

18. Ahmadraji T, Gonzalez-Macia L, Ritvonen T, Willert A, Ylimaula S, Donaghy D, et al. Biomedical Diagnostics Enabled by Integrated Organic and Printed Electronics. Anal Chem. 2017 Jul 18;89(14):7447-54.

19. Livermore DM, Day M, Cleary P, Hopkins KL, Toleman MA, Wareham DW, et al. OXA-1 $\beta$-lactamase and nonsusceptibility to penicillin/ $\beta$-lactamase inhibitor combinations among ESBL-producing Escherichia coli. Journal of Antimicrobial Chemotherapy. 2019 Feb 1;74(2):326-33.

20. Sugumar M, Kumar KM, Manoharan A, Anbarasu A, Ramaiah S. Detection of OXA-1 $\beta$-lactamase gene of Klebsiella pneumoniae from blood stream infections (BSI) by conventional PCR and in-silico analysis to understand the mechanism of OXA mediated resistance. PLoS ONE. 2014;9(3):e91800.

21. Livermore DM. beta-Lactamases in laboratory and clinical resistance. Clinical Microbiology Reviews. 1995 Oct;8(4):557-84.

22. Corrigan DK, Schulze H, Henihan G, Ciani I, Giraud G, Terry JG, et al. Impedimetric detection of single-stranded PCR products derived from methicillin resistant Staphylococcus aureus (MRSA) isolates. Biosensors and Bioelectronics. 2012 Apr;34(1):178-84.

23. Huang JM-Y, Henihan G, Macdonald D, Michalowski A, Templeton K, Gibb AP, et al. Rapid Electrochemical Detection of New Delhi Metallo-beta-lactamase Genes To Enable Pointof-Care Testing of Carbapenem-Resistant

Enterobacteriaceae. Analytical Chemistry. 2015 Aug 4;87(15):7738-45.

24. Kara P, Cavusoglu C, Cavdar S, Ozsoz M. Direct electrochemical genosensing for multiple point mutation detection of Mycobacterium tuberculosis during the development of rifampin resistance. Biosensors and Bioelectronics. 2009 Feb 24;24(6):1796-800.

25. Bard AJ, Faulkner LR. Electrochemical methods: fundamentals and applications. Second. New York: Wiley; 2001.

26. Butterworth A, Blues E, Williamson P, Cardona M, Gray L, Corrigan DK. SAM Composition and Electrode Roughness Affect Performance of a DNA Biosensor for Antibiotic Resistance. Biosensors. 2019 Feb 7;9(1):22.
27. Jiang $P$, Wang $Y$, Zhao L, Ji C, Chen D, Nie L. Applications of Gold Nanoparticles in Non-Optical Biosensors. Nanomaterials. 2018 Dec;8(12):977. 